\title{
How do predicted energy requirements compare to an armband device that indirectly measures energy expenditure in stable stroke patients?
}

\author{
S. Seery ${ }^{1}$, J. Harbison ${ }^{2}$, L. Healy ${ }^{1}$ and C. Browne ${ }^{1}$ \\ ${ }^{1}$ Department of Clinical Nutrition, St. James's Hospital, Dublin 8 and ${ }^{2}$ Department of Medical Gerontology, \\ Trinity College Dublin, Ireland
}

There is limited published research regarding energy requirements following stroke. Difficulties lie in calculating energy demands at different stages in the stroke care pathway due to factors such as the extent of stroke and changes in physical activity levels. New technologies such as the Bodymedia SenseWear ${ }^{\circledR}$ armband device (BMD) which has been evaluated against other gold standard methods ${ }^{(1)}$ are non-invasive, easy to wear and offer opportunities to more accurately determine energy expenditure in a hospital setting.

An observational study was conducted on 30 stroke patients. The population included mainly ischaemic strokes (77\%). The median age was 75 years and the majority were men $(n=18)$. Energy expenditure was indirectly measured using the BMD over 24hours. The HarrisBenedict $(\mathrm{HB})^{(2)}$ and Schofield ${ }^{(3)}$ predictive energy calculations were compared with the BMD results. Agreement was defined as within $100 \mathrm{kcals}(+/-)$ of the BMD result. Data on anthropometry, functional and nutritional status was collected and patients were screened for hyper-metabolism.

Schofield BMR had the highest level of agreement with BMD overall (50\%) while HB basal energy expenditure (BEE) had $43 \%$ agreement. When an activity factor was added to Schofield, agreement with BMD reduced further. The average metabolic equivalents ratio (METs) ranged from $0.74-1.27$. A METs of $\leq 1.0$ corresponds to sedentary/resting activities ${ }^{(4)}$ The table below shows the level of agreement based on functional status. The majority of stroke patients had a normal BMI and $63 \%$ were at medium to high risk of malnutrition as defined by 'MUST'. Only $17 \%$ required enteral tube feeding, the remaining patients tolerating normal (33\%) or modified consistency diet $(50 \%)$ with $60 \%$ prescribed oral nutritional supplements.

\begin{tabular}{|c|c|c|c|}
\hline $\begin{array}{l}\text { Rankin Modified Scale }{ }^{(4)}(\mathrm{MRS}) \\
\text { Scale of Functional ability }\end{array}$ & $\begin{array}{l}\text { Schofield } \\
\text { (BMR) }\end{array}$ & $\begin{array}{c}\text { Schofield plus } \\
\text { Activity Factor }\end{array}$ & $\begin{array}{l}\text { Harris-Benedict } \\
\text { (BEE) }\end{array}$ \\
\hline $\begin{array}{l}\text { MRS score }=2-4 \\
\text { Slight to moderate disability }(n=17)\end{array}$ & $53 \%$ & $24 \%$ & $35 \%$ \\
\hline $\begin{array}{l}\text { MRS score }=5 \\
\text { Severe disability/bedbound }(n=13)\end{array}$ & $46 \%$ & $23 \%$ & $54 \%$ \\
\hline
\end{tabular}

Low levels of agreement with the BMD may be attributed to the low levels of physical activity in this population and an overestimation of activity factors applied. Further research with larger groups using validated methods of measuring energy expenditure are required before this technology can be used in a clinical hospital setting. This study also highlights the level of nutritional risk in the stroke rehabilitation population and the reliance on nutrition support interventions.

1. Andre D \& Pelletier R et al. The Development of the SenseWear ${ }^{\mathbb{R}}$ armband, a Revolutionary Energy Assessment Device to Assess Physical Activity and Lifestyle (2006). Copyright ${ }^{\mathbb{C}}$ Bodymedia Inc. Pittsburgh, PA.

2. INDI Irish Nutrition and Dietetic Institute. Nutrition Support Reference Guide (2010).

3. Ainsworth BE et al. Medicine and Science in Sports \& Exercise (2000) 32;9:S498-504.

4. Bonita R \& Beaglehole R "Modification of Rankin Scale: Recovery of motor function after stroke.” Stroke 1988 Dec;19(12):1497-1500. 